\title{
POSTER
}

\section{Séquelles des cellulites cervico-faciales graves d'origine dentaire : Etude rétrospective et prospective}

\section{Aldosa $\mathrm{J}^{1}$, Gangloff $\mathrm{P}^{2}$, Gallet $\mathrm{P}^{3}$}

1. Assistant hospitalo-universitaire au CHU de Montpellier. Pratique privée polyclinique Saint Roch à Montpellier. Ancien interne des hôpitaux, spécialiste qualifié en chirurgie orale

2. Praticien hospitalier au CHR de Metz-Thionville. Ancien assistant hospitalo universitaire, ancien interne des hôpitaux, spécialiste qualifié en chirurgie orale

3. Praticien hospitalier universitaire au CHU de Nancy. Ancien interne des hôpitaux. Chirurgien ORL

\section{Introduction}

Les cellulites cervico-faciales d'origine dentaire sont des infections des tissus profonds de la face et du cou. Elles peuvent entraîner de nombreuses complications loco régionales potentiellement létales (médiastinite, empyème pleural, péricardite, thromboses vasculaires, choc septique, obstruction des voies respiratoires). La prise en charge médicochirurgicale de ces complications est longue et invasive. Les séquelles postopératoires peuvent être fortement handicapantes. Des études ont été menées sur les causes des cellulites cervico faciales, ou sur leurs facteurs favorisants. Le but de cette étude est d'analyser l'épidémiologie, de quantifier les séquelles et les complications liées aux cellulites cervico-faciales.

\section{Matériel et méthode}

Une étude rétrospective et prospective a été mise en place. Les patients hospitalisés suite à une cellulite cervico-faciale aiguë d'origine dentaire ont été inclus. La période d'inclusion était de mai 2012 à décembre 2014. L'étude a été menée dans les services de chirurgie maxillo faciale (CMF) et d'oto-rhino-laryngologie (ORL) du CHU de Nancy. Les autres étiologies ont été exclues. Les données cliniques et radiologiques des cellulites ont été analysées. La prise en charge et les suites opératoires ont été décrites. Des questionnaires mesurant la qualité de vie et l'impact de la pathologie ont été envoyés aux patients inclus.

\section{Résultats}

63 dossiers ont été inclus : 39 femmes et 24 hommes âgés de 42 ans en moyenne. Les patients avaient moins de 6 dents cariées ou absentes pour $75 \%$ d'entre eux. 18 patients étaient fumeurs et 20 atteints d'une pathologie chronique ou d'immunodépression. 39 cas avaient été exposés aux anti-inflammatoires avant leur admission. $32 \%$ des cellulites ont diffusé en direction cervicale et $2 \%$ en direction médiastinale. $65 \%$ des patients ont été opérés, au total 53 interventions ont été pratiquées. $11 \%$ des patients ont présenté des séquelles opératoires. Aucun décès n'a été observé. $44 \%$ des patients ont répondu à nos questionnaires de qualité de vie. 


\section{Discussion}

L'analyse des données cliniques montre que les patients atteints de formes graves ont été plus exposés aux anti-inflammatoires que les autres et ont une valeur de la PCR plus élevée, malgré des résultats non significatifs. Les patients avec des co-morbidités, fumeurs, avec une cellulite grave et avec une prise en charge plus invasive ont significativement plus de risques de développer des séquelles post opératoires. L'analyse des questionnaires de qualité de vie montre que leur pathologie a eu un impact important sur l'alimentation, la mastication et sur la douleur ressentie. Les résultats n'étaient pas corrélés avec la sévérité de la cellulite. 